\title{
Chorioamnionitis in pregnancy: a comparative study of HIV- positive and HIV-negative parturients
}

\author{
Amaka N Ocheke ${ }^{1}$, Patricia A Agaba ${ }^{2}$, Godwin E Imade ${ }^{1}$, Olugbenga A Silas ${ }^{3}$, Olanrewaju I \\ Ajetunmobi ${ }^{3}$, Godwins Echejoh ${ }^{3}$, Clement Ekere ${ }^{1}$, Ayuba Sendht ${ }^{1}$, James Bitrus ${ }^{1}$, \\ Emmanuel I Agaba ${ }^{4}$, and Atiene S Sagay ${ }^{1}$ \\ ${ }^{1}$ Department of Obstetrics and Gynaecology, University of Jos/Jos University Teaching Hospital, \\ Jos, Nigeria \\ ${ }^{2}$ Department of Family Medicine, University of Jos/Jos University Teaching Hospital, Jos, Nigeria \\ ${ }^{3}$ Department of Histopathology, University of Jos/Jos University Teaching Hospital, Jos, Nigeria \\ ${ }^{4}$ Department of Medicine, University of Jos/Jos University Teaching Hospital, Jos, Nigeria
}

\begin{abstract}
Background-Chorioamnionitis is an important risk factor for vertical transmission of HIV/ AIDS. We compared the prevalence and correlates of chorioamnionitis in HIV-positive and HIVnegative pregnant women.

Methods-HIV-positive and -negative parturients were interviewed, examined and had their placenta examined histologically for chorioamnionitis. Data regarding HIV were also retrieved from their hospital records.
\end{abstract}

Results-A total of 298 parturients ( $150 \mathrm{HIV}$ positive and 148 HIV negative) were enrolled. The two groups were similar in socio-demographic and obstetric parameters except for age. The prevalence of HCA was $57.1 \%$ in HIV-positive women and $61.6 \%$ in HIV-negative women $(p=$ 0.43). HCA staging was associated with the number of intrapartum vaginal examinations in HIVpositive subjects and nulliparity in HIV-negative subjects. The number of intrapartum vaginal examinations and coitus in the week prior to delivery significantly affected the grade of HCA in HIV-negative subjects.

Conclusion-The prevalence of HCA in both HIV-positive and HIV-negative is high. Most variables did not affect the occurrence of histologic chorioamnionitis in both groups studied except number of intrapartum examinations, coitus in the preceding one week and nulliparity which were related to severity of the disease.

\section{Keywords}

chorioamnionitis; HIV; parturients; placenta

\footnotetext{
Reprints and permissions: sagepub.co.uk/journalsPermissions.nav

Corresponding author: Amaka N Ocheke, Jos University Teaching Hospital, PMB 2076, Jos 930001, Nigeria. amakaocheke@yahoo.com.

Declaration of Conflicting Interests

The authors declared no potential conflicts of interest with respect to the research, authorship, and/or publication of this article.
} 


\section{Introduction}

HIV/AIDS is a global problem of frightening proportions. More than $95 \%$ of HIV-infected individuals live in the developing world, predominantly in sub-Saharan Africa. Despite the fact that prevention of mother to child transmission has been depicted as a milestone in the fight against AIDS, mother to child transmission of HIV is the commonest cause of paediatric HIV/AIDS. ${ }^{1,2}$ Risk factors for perinatal HIV transmission include maternal factors like high viral load, low CD4 count, advanced disease, sexually transmitted infections, obstetric factors like prolonged rupture of membranes, use of foetal scalp electrode and chorioamnionitis. ${ }^{3-8}$ Others include infant factors like prematurity, first-born twin, breastfeeding and viral factors like co-receptor mutation and HIV genotype. ${ }^{3-8}$

Chorioamnionitis is the infection of the membranes and amniotic fluid, with clinical signs and symptoms indicative of such infection and has been reported to complicate $0.9-10 \%$ of pregnancies. ${ }^{9}$ A higher incidence of chorioamnionitis has been found in women with AIDS. ${ }^{10}$

Prevalence range of $8.8 \%, 9.8 \%, 13.1 \%$ and $36 \%$ has been reported for chorioamnionitis amongst various groups of HIV-positive women. $6,8,11,12$

Chorioamnionitis is an important risk factor for vertical transmission of HIV/AIDS in that it is a risk factor on its own and also pre-disposes to other risk factors for the perinatal transmission of HIV/AIDS. ${ }^{3-8,13}$ Complications of chorioamnionitis include preterm labour, pre-labour amniorrhexis, pre-maturity, neonatal sepsis, and some of these are all risk factors for vertical transmission of HIV. ${ }^{9,11,12,14,15}$

Diagnosis of this condition is usually clinical, hence the treatment of chorioamnionitis among the obstetric population including HIV-positive women is still limited to those with acute clinical disease, though it is an established fact that subclinical and clinical acute and chronic chorioamnionitis facilitate vertical HIV transmission. In view of the adverse association between chorioamnionitis and the vertical transmission of HIV during pregnancy, empirical treatment of all HIV-infected mothers with antibiotics during delivery has been considered by some researchers. ${ }^{16}$ Another strategy is the correction of factors associated with chorioamnionitis particularly in the setting of high HIV seroprevalence.

Despite this scenario and the high prevalence of HIV/AIDS in our environment, few studies have been done on chorioamnionitis and HIV in Nigeria. Hence, we sought to find out if there is a statistically significant difference in the prevalence of chorioamnionitis between HIV-positive and HIV-negative pregnant women and factors that may influence this difference.

\section{Materials and methods}

\section{Study area and design}

This was a cross-sectional study carried out at the delivery suite of the Jos University Teaching Hospital (JUTH), Plateau State Specialist Hospital, Bingham University Teaching 
Hospital and Faith Alive Hospital, all in Jos. These are all 'President's emergency plan for AIDS relief' (PEPFAR) sites. The laboratory examinations were carried out at the histopathology laboratory of JUTH, Jos.

\section{Study subjects}

The study subjects were recruited consecutively as they presented to the delivery suites of the respective hospitals. The study participants were all consenting HIV-positive parturients and HIV-negative parturients to serve as controls. The study subjects had their placenta biopsied immediately after delivery and evaluated for chorioamnionitis.

\section{Sample size determination}

The sample size for the study was determined using a prevalence of chorioamnionitis among pregnant women of $10 \% .{ }^{9}$ This yielded a sample size of $140 \mathrm{HIV}$-positive and $140 \mathrm{HIV}$ negative parturients. However, $148 \mathrm{HIV}$-negative and $150 \mathrm{HIV}$-positive pregnant women were eventually recruited for the study.

\section{Data collection}

A questionnaire was administered to all the participants recruited for the study to obtain biodata and obstetric history. Other relevant maternal obstetric information were obtained from the participants' case notes and included duration of rupture of membrane, number of intrapartum vaginal examinations, duration of labour, route of delivery and post-partum maternal stay calculated from time of delivery to discharge.

The participants were also examined for fever (temperature $\geq 37.2^{\circ} \mathrm{C}$ ), uterine tenderness, maternal tachycardia ( $\geq 100$ beats per min), foetal tachycardia ( $\geq 160$ beats per min) and foul-smelling amniotic fluid.

Placenta from each participant was examined fresh immediately after delivery. Placental samples were taken from the centre close to the site of umbilical cord insertion, midway between cord insertion and placental margin and from the periphery of the placenta each of which included the centre of the placental lobule, chorionic plate and decidual floor. A piece of rolled up extraplacental membrane was also taken for examination. These samples were then fixed in formalin (10\% NBF) for at least $24 \mathrm{~h}$.

Histologic findings of all placentae were recorded using standardised protocol. Histologic examination was done by two pathologists who were blind to participants' HIV status.

\section{Histological examination of placenta}

Sections of these specimens were cut and placed in tissue cassettes, subsequently passed through routine histological processing, i.e. through series of reagents (formaldehyde, alcohol, wax and xylene) and processed into paraffin-embedded tissue blocks. Sections 3-4 $\mu \mathrm{m}$ thick were cut from the paraffin-embedded blocks and placed on glass slides. These were then stained with haematoxylin and eosin and subsequently examined by routine light microscopy. 


\section{Diagnosis of chorioamnionitis}

The diagnosis of chorioamnionitis was based on the modified criteria reported by Naeye et al. ${ }^{17}$ Histologic chorioamnionitis was identified as inflammatory infiltrate of neutrophils at two or more sites in the chorionic plate and extra placental membranes in all the placental sections. This inflammatory process was classified both by stage and grade. In stage 1 , the neutrophils were attached to the underside of placental plate. Stage 2 was identified when there was extension of neutrophils into the plate while in stage 3 , the neutrophils extended to the amnion. The grade of the chorioamnionitis was determined by the number of neutrophils attached to or infiltrating the plate. In grade 1 , there were $<10$ neutrophils per microscopic high-power field; in grade 2, 11 to 30 neutrophils and in grade 3, > 30 neutrophils.

\section{Data analysis}

Data analysis was done using EPI info version 3.3.2 statistical software. Continuous variables were presented as means $\pm \mathrm{SD}$ while discrete variables as proportions. The Student's t-test was used to compare group means of uniformly distributed continuous variables. The non-parametric test, Mann-Whitney U, was used to compare non-uniformly distributed continuous variables. The Pearson's Chi-Squared was used to compare proportions. Fisher Exact was used when cells had less than five observations. $p$ values < 0.05 were considered significant.

\section{Ethical considerations}

The study was approved by the Human Research Ethics Committees of the participating hospitals for the study. Informed consent was obtained from the patients prior to administration of the questionnaires, examination and specimen collection. Data were anonymised and kept secure for analysis.

\section{Results}

\section{Characteristics of study subjects}

A total of 298 parturients ( $150 \mathrm{HIV}$ positive and $148 \mathrm{HIV}$ negative) were enrolled from the labour ward of four large hospitals that provide PMTCT services in Jos metropolis. All subjects had complete data for analysis.

The characteristics of the study population are as shown in Table 1. The mean age of HIVpositive women was $31 \pm 4$ years, while that of HIV-negative women was $28 \pm 6$ years ( $p<$ 0.0001). The socio-demographic and obstetric features of the HIV-positive and -negative subjects were comparable. Only three of the HIV-positive subjects were not on antiretrovirals.

None of the patients had fever or uterine tenderness and only one patient (with HIV) had foul-smelling liquor. Only three HIV-positive women and two HIV-negative women with pre-labour rupture of membrane (PROM) received antibiotics.

Majority of the patients could not reliably determine duration of labour as their estimates ranged from 20 min to 1 week 


\section{Prevalence of chorioamnionitis}

The prevalence of histological chorioamnionitis was $57.1 \%$ among HIV-positive women and $61.6 \%$ among HIV-negative women. However, there was a statistical difference in the grade of histological chorioamnionitis between the two groups with less severe histological chorioamnionitis being more common in the HIV-negative women. The stage of histological chorioamnionitis was similar in the two groups of women. The prevalence, staging and grading of histological chorioamnionitis in the study subjects are shown in Table 2. None of the subjects was clinically diagnosed to have chorioamnionitis.

\section{Correlates of histological chorioamnionitis in HIV-positive subjects}

The correlates of histological chorioamnionitis in HIV-positive subjects are shown in Table 2. The mean age, educational status, parity, abortions, gestational age, history of PROM, number of vaginal examinations intrapartum, condom use, coitus within one week prior to delivery, history of antibiotic use and CD4 count did not significantly affect the presence of chorioamnionitis. Subjects without chorioamnionitis had more female babies $(p=0.019)$. Similarly, the mean birth weight in those without HCA was significantly lower than those with HCA.

\section{Correlates of histological chorioamnionitis in HIV-negative patients}

The correlates of histological chorioamnionitis in HIV-negative women are shown in Table 2. The table shows that the variables of age, educational status, parity, abortions, gestational age, history of coitus in the preceding one week before delivery, history of PROM, history of labour, mode of delivery, number of intrapartum vaginal exam, sex and birth weight did affect the distribution of histological chorioamnionitis in HIV-negative women.

\section{Correlates of severity of chorioamnionitis}

Tables 3 and 4 show the severity of chorioamnionitis in HIV-positive and HIV-negative subjects, respectively. The number of intrapartum vaginal examination was associated with the staging of HCA in HIV-positive women. Intrapartum vaginal examination was also associated with grading and staging of HCA in HIV-negative women.

\section{Discussion}

The placenta has a fundamental role in the vertical transmission of HIV from mother to foetus. HIV has been associated with increased risk of chorioamnionitis, which in turn is a risk factor for mother to child transmission of HIV. The prevalence of chorioamnionitis among HIV-positive and HIV-negative pregnant women in Jos and the impact of various variables on this were assessed. This study shows that the prevalence of chorioamnionitis in the study population was high and similar in the two groups of patients (57.1\%and 61.6\% of HIV-positive and HIV-negative subjects, respectively). This finding is higher than $11.4 \%$ and $11.5 \%$ reported by Torricelli et al. ${ }^{18}$ and Cuna et al. ${ }^{19}$ but similar to the findings of Curtin et al. ${ }^{20}(57.3 \%)$. However, these studies were done in term pregnancies unlike this current study that included preterm pregnancies. 
An incidence of $8.8 \%$ of HCA has been reported from Mombassa, Kenya, among HIVpositive women. ${ }^{6}$ Other studies have reported increased incidence of chorioamnionitis amongst HIV-positive women as opposed to HIV-negative women. ${ }^{15,21}$ This however, was not the finding in this study. This may probably be due to the fact that HIV-positive pregnant women are now on HAART routinely.

None of the patients had clinical chorioamnionitis, which reflects that all the cases of chorioamnionitis were subclinical. Subclinical/histological chorioamnionitis occurs more commonly than clinical chorioamnionitis. Nevertheless, the presence of histologic chorioamnionitis does not by itself guarantee the presence of significant infectious process as has been reported from other studies. ${ }^{9,18}$ These studies showed that only a small proportion of placentae with histologic chorioamnionitis had evidence of any infection. They concluded that histological chorioamnionitis in term infants is often a result of noninfectious inflammatory process. ${ }^{9,18}$

Different factors have been known to contribute to or affect the development of chorioamnionitis in pregnancy. Chorioamnionitis has been known to occur more frequently among women of low socioeconomic status. ${ }^{22}$ The educational status of an individual has been used as a good indicator of his/her socioeconomic status. This study showed that there exists a trend towards a significant association between the presence of HCA and educational status among HIV-positive subjects. However, this observation was not reproduced among HIV-negative subjects. Many reasons could have accounted for this disparity but the association of HIV and HCA is expected as HIV is largely known to be associated with poverty. It is also possible that the relatively small samples studied could have impacted the findings of this study.

The study also did not show any relationship between parity and the presence of HCA in both HIV-positive and -negative subjects. This is similar to the findings of Mwanyumba et al. ${ }^{6}$ and Smulian et al., ${ }^{9}$ where parity did not significantly affect the presence of HCA. However, nulliparity has been reported to be associated with the development of chorioamnionitis. ${ }^{22}$ This association of nulliparity with HCA was only significant for the subjects without HIV with regard to staging of HCA in our study as shown in Table 4 ( $p=$ $0.03)$.

Histologic chorioamnionitis has been reported to be inversely proportional to gestational age. It has been implicated as a causative factor of preterm deliveries while preterm deliveries are also said to be frequently complicated by choriamnionitis. ${ }^{23}$ It is known that the incidence of chorioamnionitis increases with decreasing gestational age especially before 30 weeks. However, gestational age did not have any effect on HCA in this study. It could be because majority of the patients were term as the mean EGA of the study subjects was 39 \pm 2 weeks.

This study demonstrated that sex and weight of the baby were significantly associated with HCA in HIV-positive subjects only. From the study of Smulian et al., ${ }^{9}$ birth weight had a significant relationship with HCA but no relationship with the sex of baby. While this study showed that babies born to women with HCA were heavier than those born to women 
without, Smulian et al. ${ }^{9}$ found the converse. The conflicting reports associating sex of baby and birth weight with HCA may just be epidemiological.

Prolonged labour has been implicated as a risk factor for developing chorioamnionitis. Labour lasting $12 \mathrm{~h}$ or more has been associated with a four-fold increase in risk of chorioamnionitis. ${ }^{23}$ In this study, majority of the patients could not assess how long they had been in active phase of labour. However, the effect of labour on HCA was assessed and was found to have no significant effect. This is similar to the findings of Smulian et al. ${ }^{9}$ Also, vaginal delivery has been associated with the increased risk of developing chorioamnionitis probably because of the longer time it gives for inflammatory process to occur. ${ }^{9}$ The mode of delivery in this study did not seem to impact the presence of HCA. There was also no internal foetal monitoring done in this study for any of the patients during labour as this is not the practice in our environment. This practice has been said to further increase the risk of developing chorioamnionitis in labour. ${ }^{9,23}$

PROM has been implicated as a major factor in the causation of chorioamnionitis and this has been reported to occur twice as frequent in membranes that ruptured before the onset of labour as those that rupture after labour has started. ${ }^{24}$ In this study, PROM had no effect on HCA in both HIV-negative and -positive subjects. This finding is similar to some other reported studies. ${ }^{6,12}$ In association with PROM, more than eight vaginal examination intrapartum increases the risk of chorioamnionitis by five-fold. ${ }^{24}$ The median number of intrapartum vaginal examination in this study was 1 . However, increased number of vaginal examination was found to significantly affect the grading and staging of HCA in this study. The association of intrapartum intravaginal examination and grading/staging of HCA confirms previous reports. ${ }^{19}$

Recent coitus within one week preceding delivery has been implicated in the development of choriamnionitis. ${ }^{25}$ However, this was not the finding in this study in both the HIV-positive and -negative women. Other studies have also shown like this study that sexual intercourse did not affect the occurrence of chorioamnionitis. ${ }^{26,27}$

The presence of bacterial vaginosis and a previous history of chorioamnionitis have been associated with the development of chorioamnionitis. ${ }^{28,29}$ However, this could not be assessed in this study because patients in our environment are not routinely screened for bacterial vaginosis and chorioamnionitis.

Only eight patients in this study were on antibiotics (two HIV negative, six HIV positive) and there was no correlation to the presence/absence of HCA. The study by Smulian et al. ${ }^{9}$ did not show any correlation with the use of antibiotics and HCA. However, the number of patients on antibiotics in this study was too few to make meaningful comparison with other studies.

The severity of HIV has been associated with increased risk of developing infectious morbidities including chorioamnionitis. ${ }^{10}$ This severity of HIV infection has been measured by the CD4 count and viral load. The findings from this study did not show any increased risk of chorioamnionitis with worsening of the HIV disease as measured by CD4 count and 
viral load. However, this may be due to the fact that study population was small and also that all the patients, except three, were on HAART.

The severity of chorioamnionitis in this study was assessed by grading, which indicates severity of inflammation, and staging, which indicates the spread of the disease in the placenta. Most subjects (both HIV positive and HIV negative) with chorioamnionitis were in grades 1-2 and stages 1-2 of HCA.

The limitations encountered in the study include the fact that it was a cross-sectional study and hence could not establish causality of chorioamnionitis. Additionally, patients were not followed up so it is possible that the effect of established factors related to the development of HCA could not be documented. Also, the relatively small sample size used in the study could have impacted the findings and the utilisation of viral load and CD4 count from the patients folders may have resulted in the lack of apparent association between HCA given that these parameters measured within three months may not be a true reflection of the current CD4 count and viral load.

\section{Conclusion}

The prevalence of HCA in both HIV-positive and HIV-negative parturients is high and similar. Only the variables of sex of baby and birth weight are associated with HCA in HIVpositive women, while there were no variables associated with the occurrence of HCA HIVnegative women. Intrapartum vaginal examination was associated with HCA staging in HIVpositive women and both HCA grading and staging in HIV-negative women, while nulliparity was associated with HCA staging in HIV-negative women. Further studies are needed to assess the significance of HCA and its severity on foetal and maternal outcomes.

\section{Acknowledgments}

The content is solely the responsibility of the authors and does not necessarily represent the official views of the Fogarty International Center or the National Institutes of Health.

\section{Funding}

The authors disclosed receipt of the following financial support for the research, authorship, and/or publication of this article: The project described was supported by Award Number R24TW008878 from the Fogarty International Center.

\section{References}

1. Centers for Disease Control. [accessed 7 July 2014] www.cdc.gov/hiv/basics/index.html

2. Global report: UNAIDS report on the global AIDS epidemic, 2013. Available at: http:// www.unaids.org/sites/default/files/en/media/unaids/contentassets/documents/epidemiology/2013/ gr2013/UNAIDS_Global_Report_2013_en.pdf.

3. Bhoopat L, Khunamornpong S, Sirivatanapa P, et al. Chorioamnionitis is associated with placental transmission of human immunodeficiency virus-1 subtype $\mathrm{E}$ in the early gestational period. Mod Pathol. 2005; 18:1357-1364. [PubMed: 15846390]

4. Landeesman SH, Kalish LA, Burns DN, et al. Obstetrical factors and the transmission of human immunodeficiency virus type 1 from mother to child. N Engl J Med. 1996; 334:1617-1623. [PubMed: 8628356] 
5. Newell ML, Peckham C. Risk factors for vertical transmission of HIV-1 and early markers of HIV-1 infection in children. AIDS. 1993; 7:S591-S597.

6. Mwanyumba F, Gaillard P, Inion I, et al. Placental inflammation and perinatal transmission of HIV-1. J Acquir Immune Defic Syndr. 2002; 29:262-269. [PubMed: 11873075]

7. Temmerman M, Nyong'o A, O' Bwayo J, et al. Risk factors for mother-to-child transmission of human immunodeficiency virus-1 infection. Am J Obstset Gynecol. 1995; 172:700-705.

8. Wabwire-Mangen F, Gray RH, Mmiro FA, et al. Placental membrane inflammation and risks of mother-to-child transmission of HIV-1 in Uganda. J Acquir Immune Defic Syndr. 1999; 22:379_ 385. [PubMed: 10634200]

9. Smulian JC, Shen-Schwarz S, Vintzileos AM, et al. Clinical choriamnionitis and histologic placental Inflammation. Obstet Gynecol. 1999; 94:1000-1005. [PubMed: 10576190]

10. Holmes W. Vertical transmission of HIV. Lancet. 1991; 337:793-794. [PubMed: 1672415]

11. Van Dyke RB, Korber BT, Popek E, et al. The ariel project: a prospective cohort study of maternalchild transmission of human immunodeficiency virus type 1 in the era of maternal anti-retroviral therapy. J Infect Dis. 1999; 179:319-328. [PubMed: 9878014]

12. Ladner J, Leroy V, Hoffman P, et al. Chorioamnionitis and pregnancy outcome in HIV-infected African women. J Acquir Immune Defic Syndr Hum Retroviral. 1998; 18:293-298.

13. Chi BH, Mudenda V, Levy J, et al. Acute and chronic chorioamnionitis and the risk of perinatal human immunodeficiency virus-1 transmission. Am J Obstet Gynecol. 2006; 194:174-181. [PubMed: 16389028]

14. Naeye RL, Ross SM. Subclinical chorioamnionitis. Clin Obstet Gynaecol. 1982; 9:593-607. [PubMed: 6756749]

15. Gray GE, McIntyre JA. HIV and pregnancy. BMJ. 2007; 334:950-953. [PubMed: 17478849]

16. Taha TE, Brown ER, Hoffman IF, et al. A phase III clinical trial of antibiotics to reduce chorioamnionitis-related perinatal HIV-1 transmission. AIDS. 2006; 20:1313-1321. [PubMed: 16816561]

17. Naeye RL, Maisels MJ, Lorenz RP, et al. The clinical significance of placental villous edema. Paediatrics. 1983; 71:588-594.

18. Torricelli M, Voltolini C, Conti N, et al. Histologic chorioamnionitis at term: implications for the progress of labor and neonatal wellbeing. J Matern Fetal Neonatal Med. 2013; 26:188-192. [PubMed: 22928534]

19. Cuna A, Hakima L, Tseng Y, et al. Clinical dilemma of positive histologic chorioamnionitis in term newborn. FrontPaediatr. 2014; 2:1-5.

20. Curtin WM, Katzman PJ, Florescue H, et al. Accuracy of signs of clinical chorioamnionitis in the term parturient. J Perinatol. 2013; 33:422-428. [PubMed: 23154669]

21. Schwartz DA, Sungkarat S, Shaffer N, et al. Placental abnormalities associated with human immunodeficiency virus type 1 infection and perinatal transmission in Bangkok, Thailand. J Infect Dis. 2000; 182:1652-1657. [PubMed: 11069236]

22. Van Gelderen, CJ. Subclinical chorio- amnionitis and chorio-amnionitis. In: Cronje, HS.; Grobler, CJF., editors. Obstetrics in Southern Africa. 2nd. Pretoria: Van Schaik Publishers; 2005. p. 461-470.

23. Galinsky R, Polglase GR, Hooper SB, et al. The consequences of chorioamnionitis: preterm birth and effects on development. J Pregnancy. 2013; 2013:412831. [PubMed: 23533760]

24. Naeye RL. Factors that predispose to premature rupture of fetal membranes. Obstet Gynecol. 1982; 60:93-98. [PubMed: 7088456]

25. Naeye RL, Ross S. Coitus and chorioamnionitis: a prospective study. Early Hum Dev. 1982; 6:9197. [PubMed: 7198968]

26. Mills JL, Harlap S, Harley EE. Should coitus late in pregnancy be discouraged? Lancet. 1981; 2:136. [PubMed: 6113493]

27. Aziken ME, Orhue AA, Okpere EE, et al. Sexual intercourse and preterm delivery: any correlation. J Turkish-German Gynecol Assoc. 2007; 8:177-183.

28. Yudin MH. Bacterial vaginosis in pregnancy: diagnosis, screening and management. Clin Perinatol. 2005; 32:617-627. [PubMed: 16085023] 
29. Laibi VR, Sheffield JS, Roberts S, et al. Recurrence of clinical chorioamnionitis in subsequent pregnancies. Obstet Gynecol. 2006; 108:1493-1497. [PubMed: 17138785] 


\section{Table 1}

Socio-demographic, obstetric and clinical features of subjects.

\begin{tabular}{|c|c|c|c|}
\hline Variable & HIV-positive subjects & HIV-negative subjects & $p$ \\
\hline Age (years), mean $\pm \mathrm{SD}$ & $31 \pm 4$ & $28 \pm 6$ & $<0.0001$ \\
\hline \multicolumn{4}{|l|}{ Educational status, $N(\%)$} \\
\hline None & $4(57.1)$ & $3(42.8)$ & $0.15^{a}$ \\
\hline Primary & $21(70)$ & $9(30)$ & \\
\hline Secondary & $60(46.5)$ & $69(53.5)$ & \\
\hline Tertiary & $65(49.2)$ & $67(50.8)$ & \\
\hline \multicolumn{4}{|l|}{ Occupation, $N(\%)$} \\
\hline Professional & $16(44.4)$ & $20(55.6)$ & 0.39 \\
\hline Skilled labour & $21(52.5)$ & $19(47.5)$ & \\
\hline Unskilled labour & $63(54.8)$ & $52(45.2)$ & \\
\hline Unemployed & $50(46.7)$ & $57(53.3)$ & \\
\hline Gestational age (weeks), mean \pm SD & $38 \pm 2$ & $38 \pm 2$ & 0.87 \\
\hline Parity, median (IQR) & $2(0-9)$ & $1(0-7)$ & 0.42 \\
\hline Nulliparity, $N(\%)$ & $29(37.2)$ & $49(62.8)$ & 0.008 \\
\hline Last coitus $\leq$ one week before delivery & $42(50.6)$ & $41(49.4)$ & 0.89 \\
\hline History of PROM, $N(\%)$ & $33(53.2)$ & $29(46.8)$ & 0.58 \\
\hline \multicolumn{4}{|l|}{ Mode of delivery, $N(\%)$} \\
\hline Elective CS & $22(78.6)$ & $6(21.4)$ & 0.0005 \\
\hline Emergency CS & $8(50)$ & $8(50)$ & \\
\hline SVD & $120(47.2)$ & $134(52.8)$ & \\
\hline Labour, $N(\%)$ & $128(47.4)$ & $142(52.6)$ & 0.002 \\
\hline Episiotomy, $N(\%)$ & $5(8.5)$ & $54(91.5)$ & $<0.0001$ \\
\hline Tachycardia, $N(\%)$ & $17(68)$ & $8(32)$ & 0.19 \\
\hline Foetal heart rate, mean \pm SD & $139.1 \pm 6.7$ & $138.4 \pm 10$ & 0.52 \\
\hline Vaginal tear, $N(\%)$ & $16(10.9 \%)$ & $26(17.6 \%)$ & 0.1 \\
\hline Weight of baby $(\mathrm{kg})$, mean \pm SD & $2.97 \pm 0.52$ & $3.06 \pm 0.50$ & 0.16 \\
\hline Placental weight $(\mathrm{g})$, mean $\pm \mathrm{SD}$ & $535.2 \pm 138.3$ & $572 \pm 126.3$ & 0.05 \\
\hline
\end{tabular}




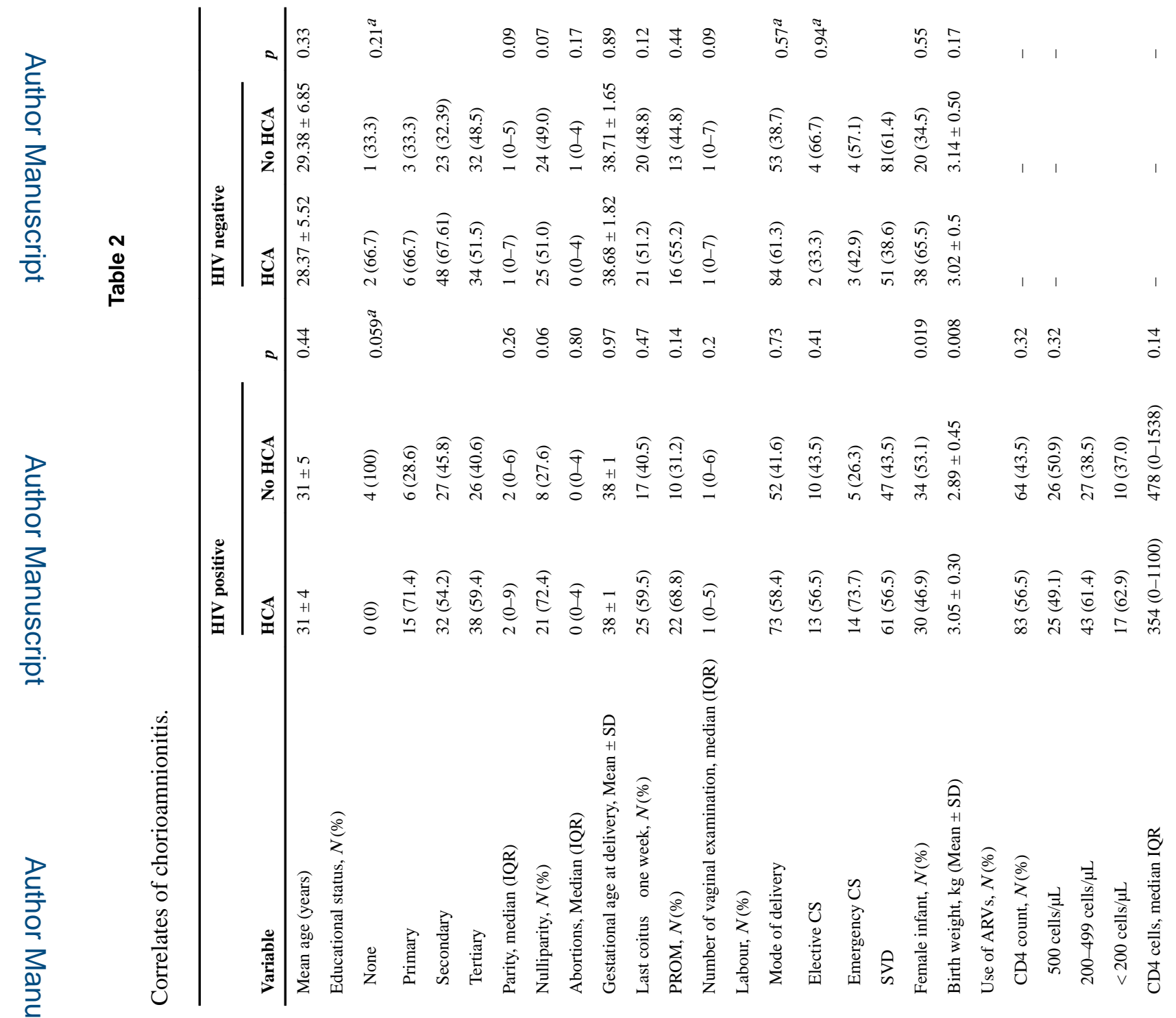




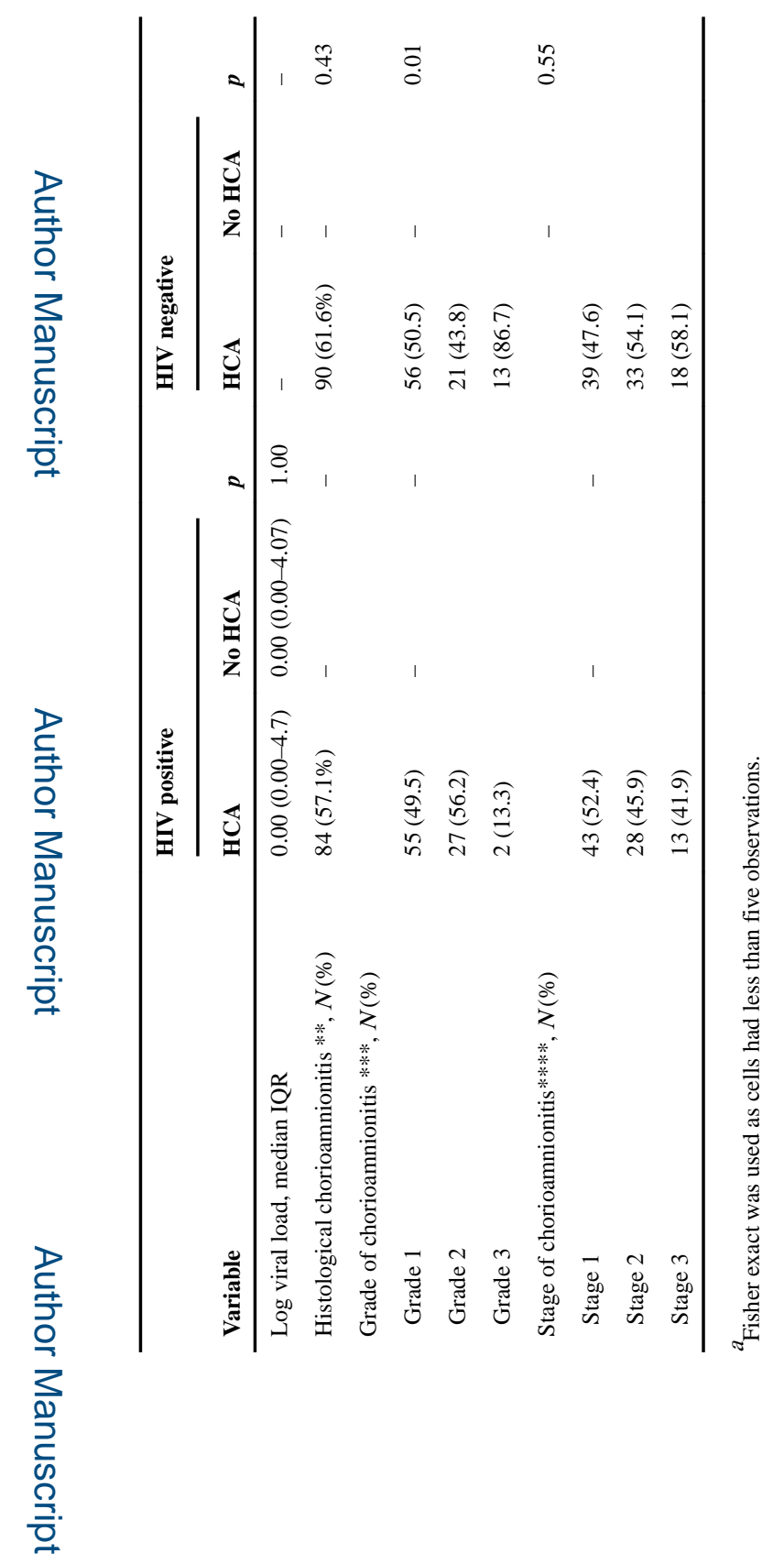

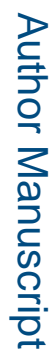

Int J STD AIDS. Author manuscript; available in PMC 2016 April 01. 


\section{을 \\ 졸}

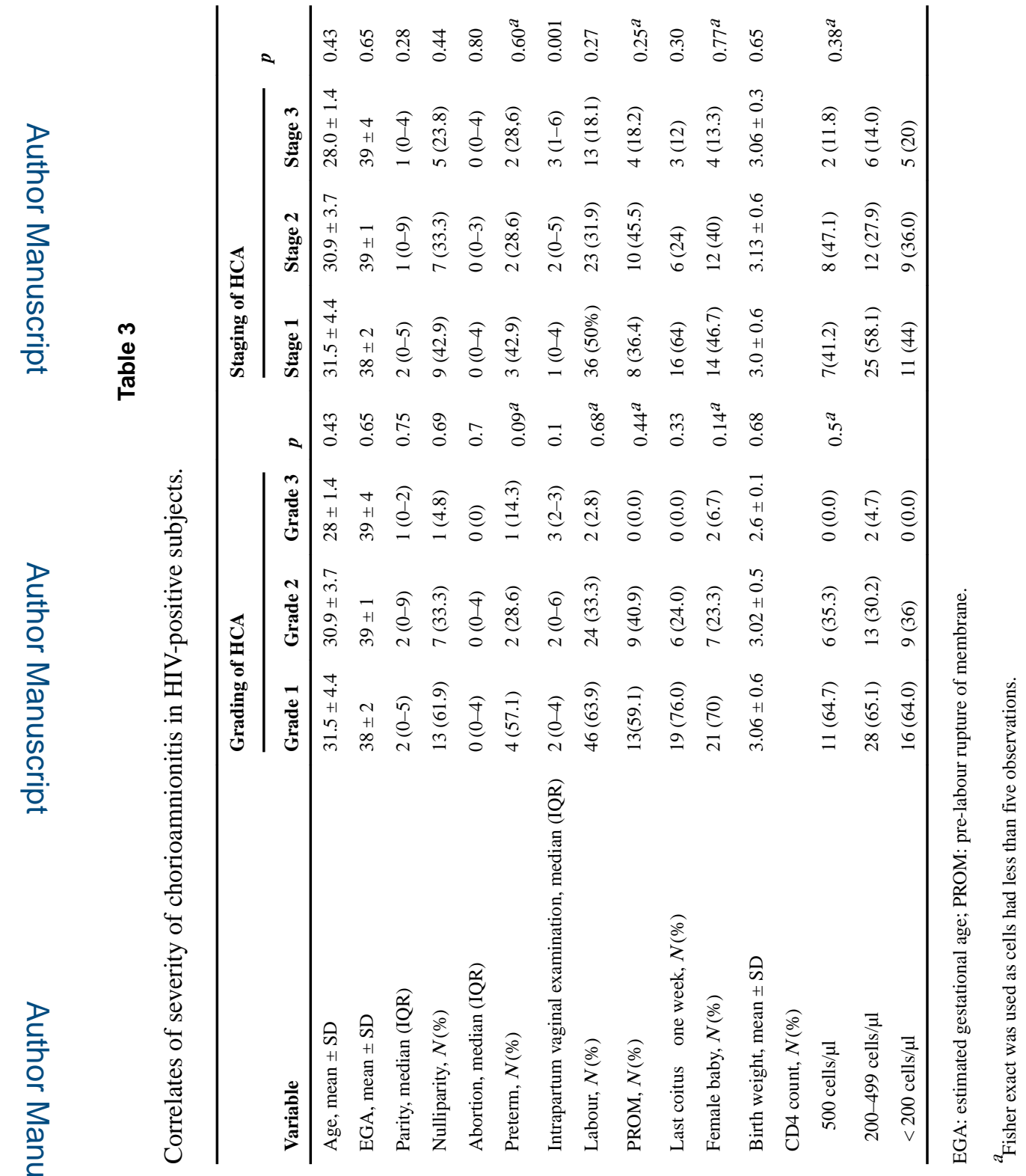




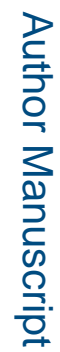

을

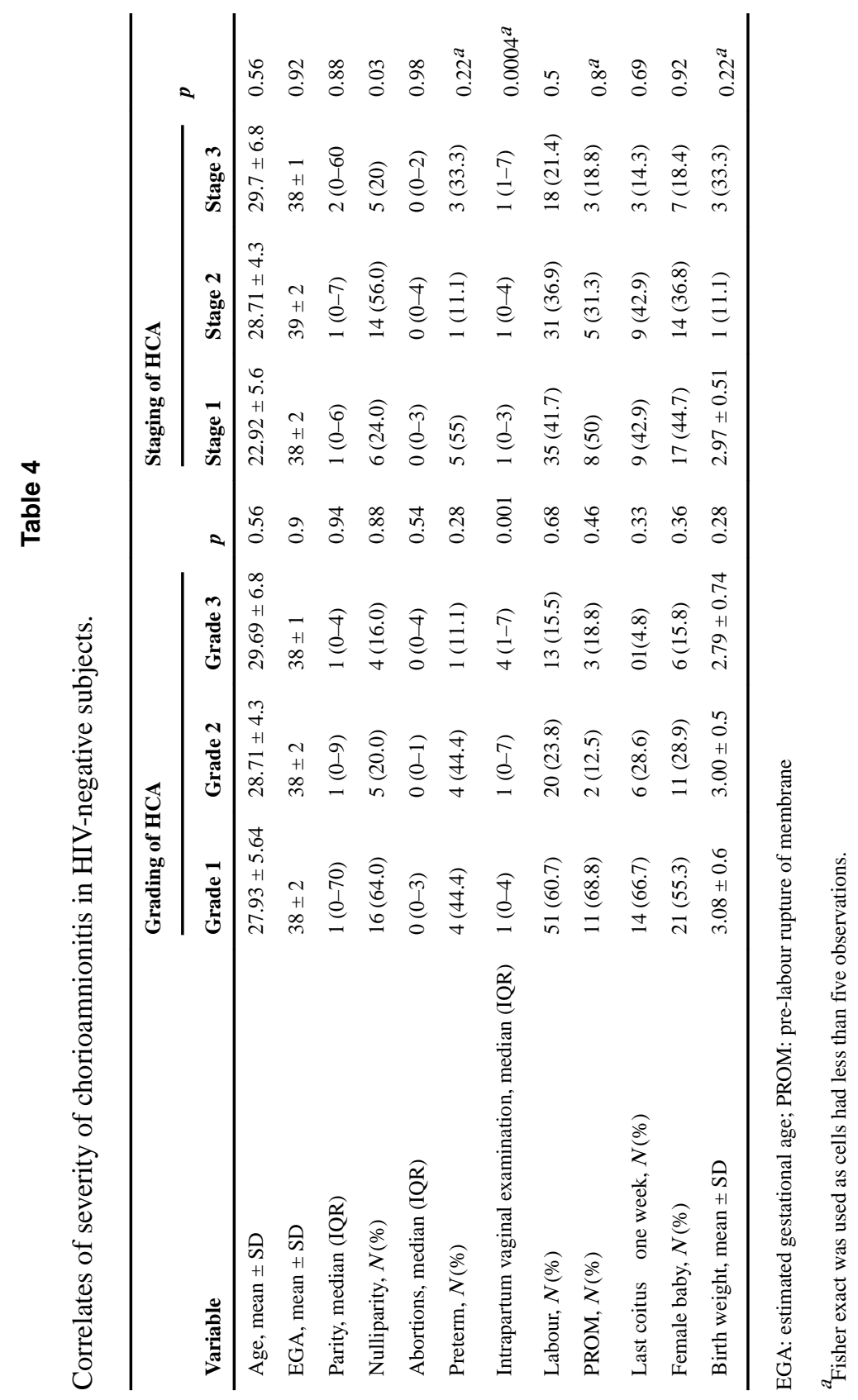

